\title{
MICRO-TOMOGRAPHY USING SYNCHROTRON RADIATION
}

Q. C. Johnson, J. H. Kinney, U. Bonse,

M. C. Nichols, R. Nusshardt, and J. M. Brase

This paper was prepared for subnittal to Materials Research Society

Spring Meeting

Palo Alto, CA

April $15-17,1986$

April 9, 1986

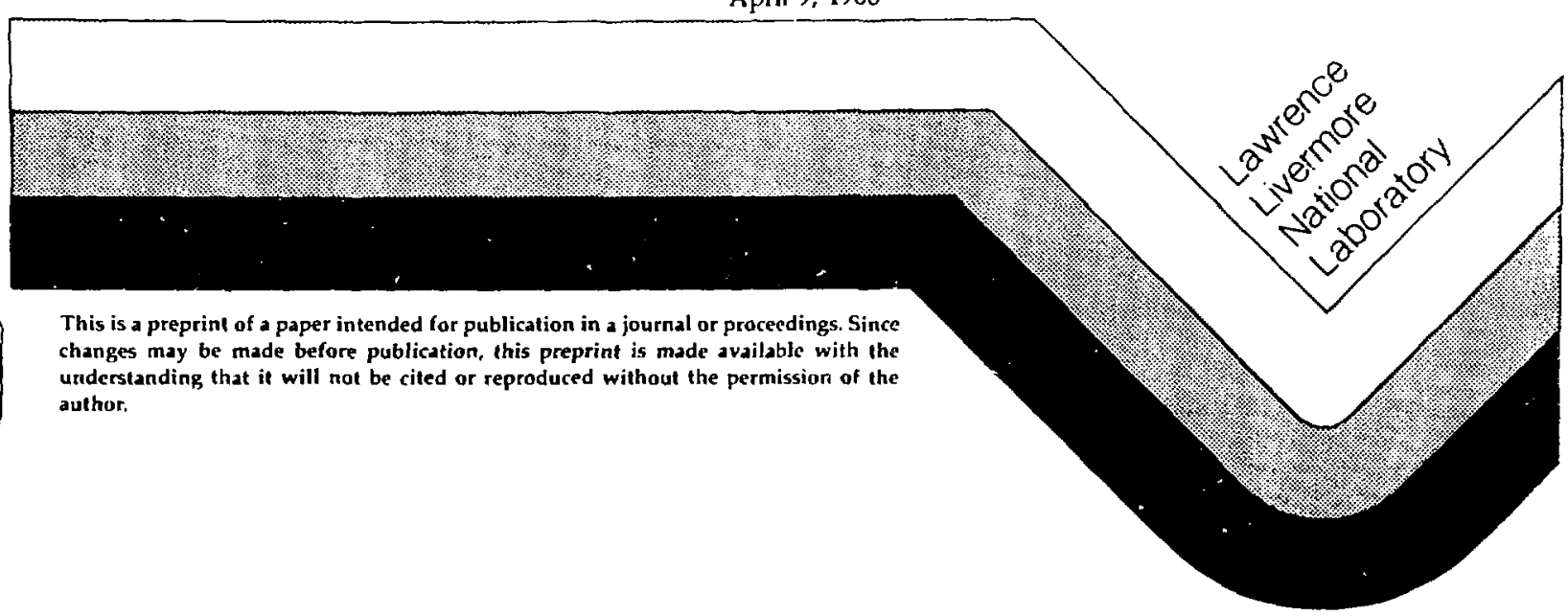




\title{
MICRO-TOMOGRAPHY USING SYNCHROTRON RADIATION ${ }^{\dagger}$
}

\author{
QUINTIN C. JOHNSON*, JOHN H. KINNEY*, ULRICH BONSE**, MONTE C. NICHOLS,***
RUDOLF NUSSHARDT** AND JAMES M. BRASE* \\ * Lawrence Livermore National Laboratory, Livermore, CA 94550 \\ ** Dortmund University, Dept. of Physics, Dortmund, West Germany \\ *** Sandia National Laboratory, Livermore, CA 94550
}

\begin{abstract}
This paper discusses the results of recent experiments at Stanford Synchrotron Radiation Laboratory (SSRL) and Hamburger Synchrotronstrahlungslabor (HASYLAB) which were designed to explore the feasibility of using synchrotron radiation in high-resolution, computerized, critical-absorption tomography. The results demonstrate that it is possible, using absorption-edge differencing, to identify adjacent elements in the periodic table with high sensitivity. Furthermore, by using the fine structure in the absorption spectra, it is possible to distinguish between regions of different chemical states. Methods of using synchrotron radiation for high-resolution, three-dimensional chemical-state mapping in small samples are discussed.
\end{abstract}

\section{INTRODUCTION}

$X$-ray computed tomography (CT) is a potentially valuable tool for materials characterization. The large depth of $\mathrm{x}$-ray penetration allows $\mathrm{CT}$ to characterize internal structures in macroscopic (1 $\mathrm{cm}$ ) objects with little sample preparation; whereas, electron microscopy, with its inherently greater spatial resolution, can only provide threedimensional information on very thin, well prepared materials. $X$-ray imaging (in particular $C T$ ) complements electron imaging by providing, nondestructively, information about materials which cannot be obtained in any other manner.

Unfortunately, limitations on conventional $x$ ray sources and detectors make it prohibitive to use CT to resolve structures smaller than a $\mathrm{mm}$ in spatial dimension. Therefore, CT applications to materials questions outside of medical diagnostic radiology and inspection of industrial products has not occurred.

Synchrotron radiation (SR), because of its high flux and low divergence, is ideally suited for applications in CT. Also, because SR has a continuous energy spectrum, the source can be tuned with a very narrow bandwidth $\left(\triangle E / E<10^{-3}\right)$. This makes it possible to use critical absorption and fine-structure differencing for mapping the location of elements and their chemical states in small samples.

'Work performed undet the auspices of the U.S. Deparment of Energy by Lawrence Livermore National Laboratory under contrart No. W-7504-ENG-48.
This paper is divided into three sections:

(1) Provides a brief introduction to the problem of reconstructing three-dimensional images from their $x$-ray transmission projections, describes the principal sources of error, and shows that many putential problems in micro-tomography can be avoided by using SR in place of conventional $x$ ray sources; (2) Discusses the technical considerations involved in planning and executing a tomographic inspection on a synchrotron source; and, (3) Describes, within the context of micro-tomography, preliminary observations which demonstrate inherent advantages of imaging with SR.

\section{BACKGROUND}

The goal in transmission CT is to be able to map cross sections of an object from measured projection data; i.e., determine the values of $\mu(x, y, z)$, the $x$-ray attenuation coefficient, at specified points within an observed volume. The projection data, which is the transmitted intensity reaching the detector, is given for a polychromatic $x$-ray source by

$$
I=\int S(E)\left[\exp -\int \mu(x, y, z, E) d l\right] d E,
$$

where $S(E)$ is the energy spectrum of the source and $\mu(x, y, z, E)$ is the energy-dependent attenuation coefficient at a point on the projection. The integral within the brackets is the line integral 
over the path from the source to the detector. For a nearly monochromatic synchrotron beam, $S(E)$ is well approximated by $S(E)=I_{0} \delta\left(E-E_{0}\right)$, and Eq.(1) becomes

$$
\mathrm{I}=\mathrm{I}_{\mathrm{o}}\left[\exp -\int \mu\left(\mathrm{x}, \mathrm{y}, \mathrm{z}, \mathrm{E}_{\mathrm{o}}\right) \mathrm{dl}\right] .
$$

The natural $\log$ of Eq. (2) gives

$$
\ln \left(\mathrm{I}_{\mathrm{o}} / \mathrm{I}\right)=\int \mu\left(\mathrm{x}, \mathrm{y}, \mathrm{z}, \mathrm{E}_{\mathrm{o}}\right) \mathrm{dl},
$$

which is the familiar form of the Radon transform. ${ }^{1}$ The Radon transform associates a function in coordinate space with another function in a line-integral space. The objective in $\mathrm{CT}$ is to numerically estimate the inverse Radon transform from a finite set of projection data.

The problem of evaluating the inverse Radon transform has been heavily discussed in the literature. For background, however, we will discuss the major sources of error which can lead to artifacts in the reconstructed image. Furthermore, we will show that a synchrotron source is capable of removing at least two of these major sources. Errors in reconstruction can arise from ${ }^{2}$
a. photon statistics (noise).
b. beam-hardening effects.
c. partial volume effects.
d. detertor non-linearities.
e. mechanical instabilities.

Of these, the noise associated with an insufficient number of counts is perhaps the most significant.

Grodzins $^{3}$ has developed a simple relationship between the incident $x$-ray fluence on the sample and the signal-to-noise ratio. To observe differences in $\mu$ between neighboring voxels (volume elements) as small as $1 \%$, the necessary $x$-ray fluence on the sample must be

$\Phi\left(\frac{\text { photons }}{\text { pixel area }}\right)=\frac{2 \times 10^{4} D \exp (\mu \mathrm{D})}{\mathrm{w}^{3} \mu^{2}}$,

where $D$ is the sample diameter and $w$ is the pixel width. The optimum $x$-ray energy for attenuation measurements is that which gives $\mu=2 / D$. Using this in Eq. (4) yields

$$
\Phi=4 \times 10^{4}(\mathrm{D} / \mathrm{w})^{3} .
$$

Equation 5 indicates that the fluence requirements increase as the third power of the ratio of the sample diameter to pixel width. For a $0.5-\mathrm{cm}$ sample and a $50-\mu \mathrm{m}$ pixel width, the fluence necessary to obtain a $1 \%$ sensitivity is $\Phi \geq 4 \times 10^{10}$ photons / unit pixel area. For a $50-\mu \mathrm{m}$ pixel, this corresponds to a photon fluence of $10^{13}$ photons $/ \mathrm{mm}^{2}$. To ohtain higher resolution, either a smaller diameter sample or a longer count duration should be used.

Figure 1 shows the energy spectrum of the 54-pole wiggler at SSRL. ${ }^{4}$ For $x$-ray energies of interest in most imaging experiments, fluxes near $10^{12}$ per $0.1 \%$ energy bandwidth per $\mathrm{mm}^{2}$ can be obtained. Scan times of 10 seconds per projection are sufficient for the above example. For the approximately 150 angular rotations needed for reconstruction, nearly 30 minutes are required. Furthermore, changing the constraini from $1 \%$ to $2 \%$ sensitivity reduces the total counting time to under 10 minutes. Increases of at least an order of magnitude in the $x$-ray flux can be anticipated in the next generation of SR sources.

Beam hardening is a second source of error present in conventional $C T$. Beam hardening describes the fact that as a polychromatic $x$-ray beam passes through a sample, the low-energy component is attenuated more rapidly than the high-energy component. This shifts the spectrum to greater energies, hence the term "hardening." Beam hardening poses an image-reconstruction problem, because attenuation in a given volume element is no longer independent of the sample

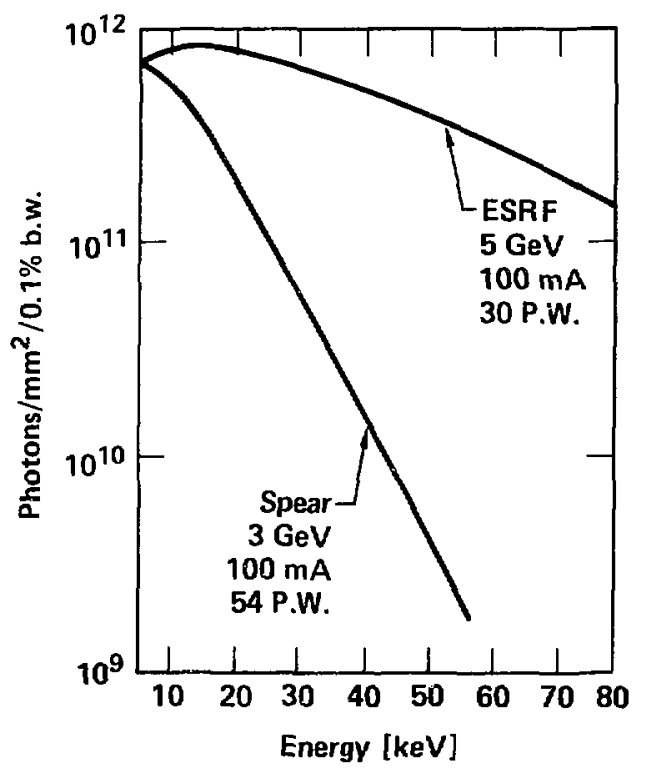

Figure 1. Energy spectra of 54 pole wiggler at SSAL and that expected for the $\mathbf{3 0}$ pole wiggler at the European Synchrotron Facility. 


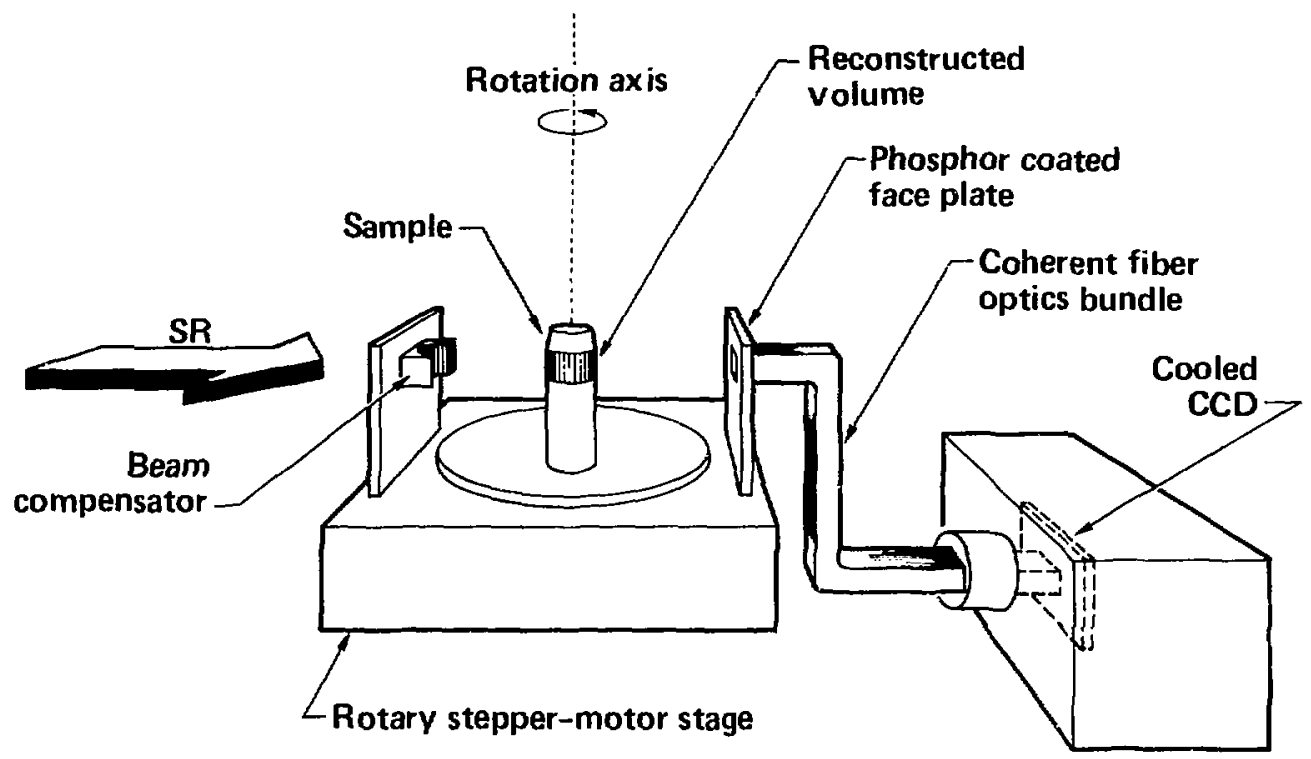

Figure 2. Apparatus for performing CT with synchrotron radiation.

orientation with respect to the beam. Mathematically, beam hardening introduces non-linearities into Eq. (3). Because synchrotron sources provide intense radiation which can be made nearly monoenergetic with chromators of high harmonic rejection, beam hardening will not be a problem in micro-tomography.

Partial volume effects describe the breakdown in the assumption that $\mathrm{d} \mu / \mathrm{d} z=0$ within a given voxel. In this notation, $z$ is normal to the plane of the slice. Partial volume effects can be reduced by decreasing the thickness of a slice so that the sampled region is more apt to be uniform.

The last two principal sources of error, detector non-linearities and mechanical instabilities, must be considered when designing a tomography experiment. The next section describes the approach we are developing for an upcoming series of micro-tomography experiments.

\section{EXPERIMENTAL APPROACH}

\section{Detector}

The half-hour exposure time, estimated for the previous example, is based upon the use of a linear detector array where all pixels in a slice are measured simultaneously. If this is not the case, and collimator pinholes are rastered across the sample, then the data collection time becomes ex- orbitant. Even if a linear array is used, however, only the examination of thin slices $(100 \mu \mathrm{m})$ would be temporally feasible. Sampling all of the voxels in a slice as thin as $1 \mathrm{~mm}$, for example, would take several hours.

We are developing a detector system which should allow us to make simultaneous measurements of up to 390 slices, each approximately 25$\mu \mathrm{m}$ thick. This means a volume of nearly a cm can be investigated in less than an hour. The heart of this system is a thermoelectrically cooled TI 4849 CCD $(390 \times 584)$ array which has $22-\mu \mathrm{m}$ square pixels. Because $C C D$ arrays cannot image energetic $x$-rays directly, the transmitted beam will be converted to visible light in a high-resolution phosphor. The visible light will be directly coupled to the CCD through a coherent fiber optics bundle. Figure 2 is a schematic showing the detector and sample arrangements.

Principal sources of error in this detector concept are noise and non-linearities. Noise is due to dark currents and readout. At the operating CCD temperature, the measured dark current is only one electron per pixel per second, and this can easily be subtracted from the signal. The readout noise is roughly 10 electrons per pixel, and since it is random, its effects can be reduced by signalaveraging techniques. ${ }^{5}$ Since each pixel is calibrated and operated as a separate detector there is no problem associated with non-linearities be- 
tween pixels. However, it is important that the individual pixel response be linear over a large dynamic range. For the TI $4849 \mathrm{CCD}$, this dynamic range is of the order $10^{5}$.

\section{Positioner}

CT relies on accurate knowledge of the path of each transmitted beam through the sample. It is imperative that the center of rotation with respect to the source and detector be known with great accuracy. It is also important that the rotation stage does not exhibit eccentric or wobbly motion. For conventional tomography, these concerns are easily managed. However, in micro-tomography, small misalignments can lead to major positioning artifacts. If a few $\mu \mathrm{m}$ resolution is desired in the reconstrurtion, then the rotational motion must be precisely defined.

In our experimental arrangement, the sample is positioned on a precise optical stage which is physically connected to the detector platform to reduce independent vibration. The precision stage is stepper-motor controlled in $0.01^{\circ}$ steps with a positioning accuracy of better than $10^{-3}$ degree. Eccentric motion is less than $1 \mu \mathrm{m}$ and the wobble is of order $10^{-7}$ radian. This is well within the tolerances needed for the resolution we will achieve with a first-generation detector.

\section{PRELIMINARY OBSERVATIONS}

\section{Chemical Sensitivity}

Attempts at using critical absorption-edge differencing to enhance contrast in radiography have been made by many investigators. In fact, the term dichromography was proposed by Jacobson as early as 1953 to describe two-color imaging using conventional sources, ${ }^{6}$ With an intense SR source and modern digital-imaging techniques, it is possible to go beyond these early attempts at contrast euhancement and to quantitatively perform elemental and chemicalstate mapping non-destructively.

In a recent series of experiments at SSRL and HASYLAB, we investigated the chemical sensitivity obtained by using absorption-edge and finestructure differencing. Figure $3(a)$ shows a polychromatic radiograph of overlaid meshes of $\mathrm{Cu}$ and $\mathrm{Ni}$. The electroformed $\mathrm{Cu}$ mesh was $250 \mu \mathrm{m}$ center to center with a width of $15 \mu \mathrm{m}$. The $\mathrm{Ni}$ mesh was $125 \mu \mathrm{m}$ center to center with a width of $15 \mu \mathrm{m}$. Both meshes were $2.5-\mu \mathrm{m}$ thick. A sequence of absorption images was taken at a few

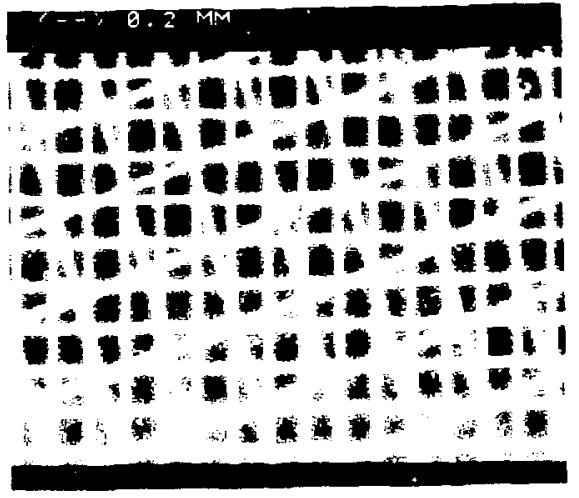

(a)

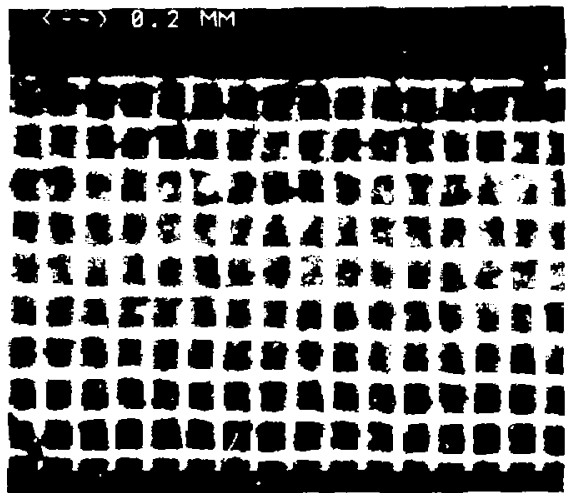

(b)

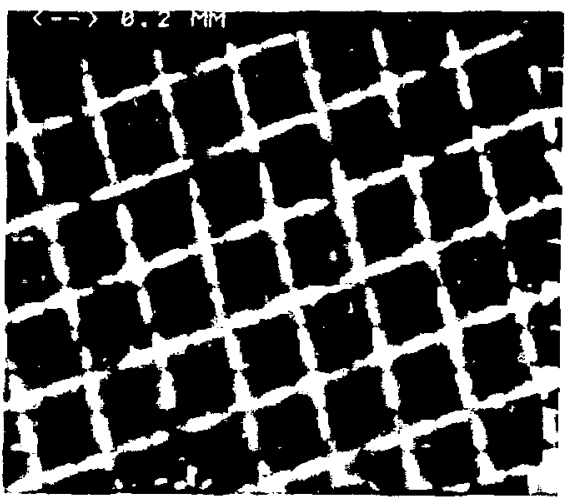

(c)

Figure 3. (a) Typical polychromatic radiograph showing overlapping $\mathrm{Cu}$ and Ni meshes; (b) difference image taken above and below the $\mathrm{Ni} \mathrm{K}$-edge; and, (c) difference image taken above and below the Cu Kedge. 
$\mathrm{eV}$ above and below the $\mathrm{Ni}$ and $\mathrm{Cu}$ K-edges. This sequence was digitized and subtracted, and the resulting images are shown in Figures 3(b) $\mathrm{Ni}$ and 3 (c) $\mathrm{Cu}$. The separate meshes are clearly contrasted, even though the elements are adjacent to each other in the periodic table and the material thicknesses are only a few $\mu \mathrm{m}$.

From these and other radiographs, we determined that a sensitivity of $0.1 \mathrm{mg} / \mathrm{cm}^{2}$ can be obtained using synchrotron radiation. This is an order of magnitude better than can be obtained using a conventional $x$-ray source. The elemental sensitivity that can be obtained in a three-dimensional mapping depends critically on the sample size and composition. Grodzins ${ }^{7}$ has estimated that concentrations as low as 10 to $100 \mathrm{ppm}$ can be detected with $1-\mu \mathrm{m}$ spatial resolution under optimum conditions using synchrotron radiation. Even lower concentrations can be mapped if the resolution requirements are relaxed or the $x$-ray fluence is increased.

As a further demonstration of the potential of CT with SR, we studied the possibility of using the fine-structure in the absorption edge to map regions of different chemical states. In the nearedge region, the absorption coefficient often is significantly affected by the chemical state of an element. Figure 4 shows where we have measured the K-edge XANES for $\mathrm{Fe}$ and $\mathrm{Fe}_{2} \mathrm{O}_{3}$. In this system, a shift in the K-edge energy of several $\mathrm{eV}$ is observed between the pure element and its oxide. We wished to determine if this shift could be exploited to identify and enhance the radiographic contrast of the oxide in the presence of the pure metal.

We imbedded fine powders of ultra-pure $\mathrm{Fe}(<50 \mu \mathrm{m})$ and $\mathrm{Fe}_{2} \mathrm{O}_{3}(<100 \mu \mathrm{m})$ in 1-mm thick epoxy matrixes. Out of these matrixes, adjacent wedges of $\mathrm{Fe}$ and the oxide were constructed which were approximately $5-\mathrm{mm}$ wide and had a slight taper which guaranteed transmission of the $x$-ray beam within the field of view. A series of absorption images was taken at the energies shown in Fig. 4. In Fig. 5(a), the wedges of Fe (top) and $\mathrm{Fe}_{2} \mathrm{O}_{3}$ (bottom) powders are imaged with $\mathrm{x}$ rays at energy $E_{1}$. Figure $5(\mathrm{~b})$ shows the result of digitally subtracting absorption images taken at $E_{2}$ and $E_{3}$. The change in contrast is sufficient to eliminate all artifacts of the oxide and leave behind a clear image of the pure iron matrix.

Figures 3 and 5 clearly demonstrate that the tunability of the SR can be exploited to enhance the contrast of one chemical state over another, and that it may be possible to generate quantitative three-dimensional elemental and chemical

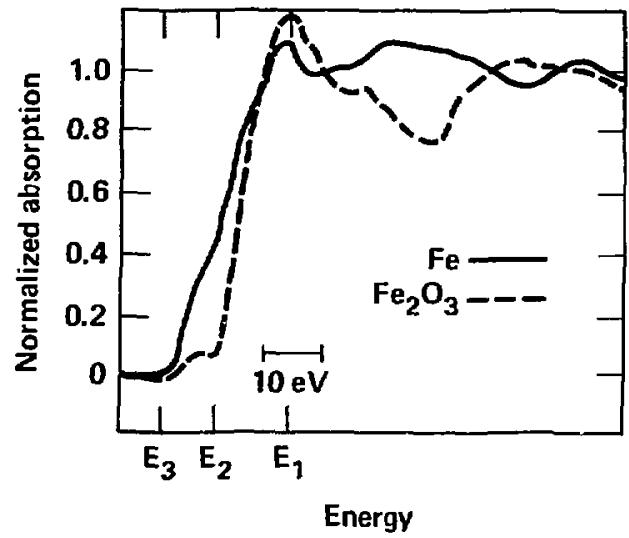

Figure 4. Absorption edge tine structure for $\mathrm{Fe}_{\mathrm{\theta}}$ and $\mathrm{Fe}_{2} \mathrm{O}_{3}$ taken at HASYLAB. The energies marked on the abscissa are the points where absorption images were obtained.

maps of small samples. This capability should prove useful in the materials and biological sciences.

\section{Resolution of $C T$ with $S R$}

The high-contrast resolution of a CT system is defined by its modulation transfer function (MTF) ${ }^{B}$ In our proposed system, the MTF will be a product of individual MTFs for the phosphor, fiber optics, and the CCD array. Though this has not yet been measured for our contiguration, we have made a conservative model of the MTF for calculational purposes to demonstrate the advantages of CT with SR over conventional techniques.

We created a phantom object which is $0.6 \mathrm{~cm}$ in diameter and consists of RT3075 epoxy in which small air-filled holes have been made. These holes range in diameter from $50 \mu \mathrm{m}$ to $200 \mu \mathrm{m}$. Figure 6 shows the configuration of the holes in the phantom.

The x-ray transmission through the phantom was calculated with a Monte-Carlo ${ }^{9}$ code which allowed for scattering and fluoresence. A detector was placed $1 \mathrm{~cm}$ from the outer edge of the sample. The simulated $x$-rays were monoenergetic $(10 \mathrm{keV})$, and had a divergence similar to that at a synchrotren source $\left(10^{-4}\right.$ radians). $X$-ray transmission was calculated for 60 angles, equally spaced between 0 and $180^{\circ}$.

The calculated projection data were reconstructed using a convolution method with a Shepp-Logan filter and the resulting cross section is shown in Fig. $7 .^{10}$ Detailed analysis of the computer simulation indicates that for the $200-\mu \mathrm{m}$ 


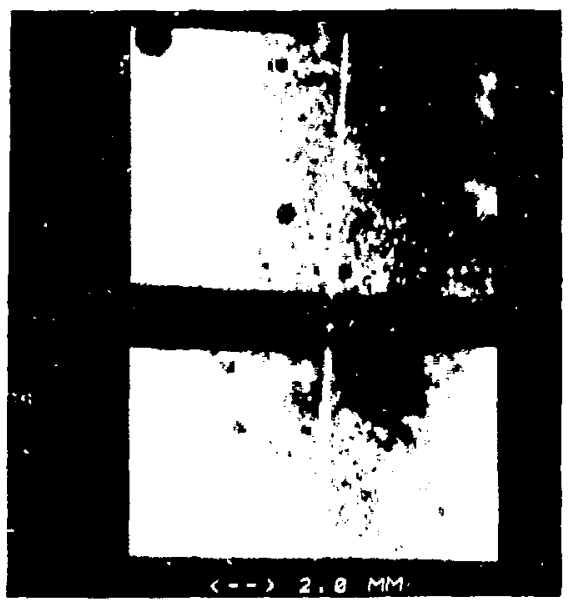

(a)

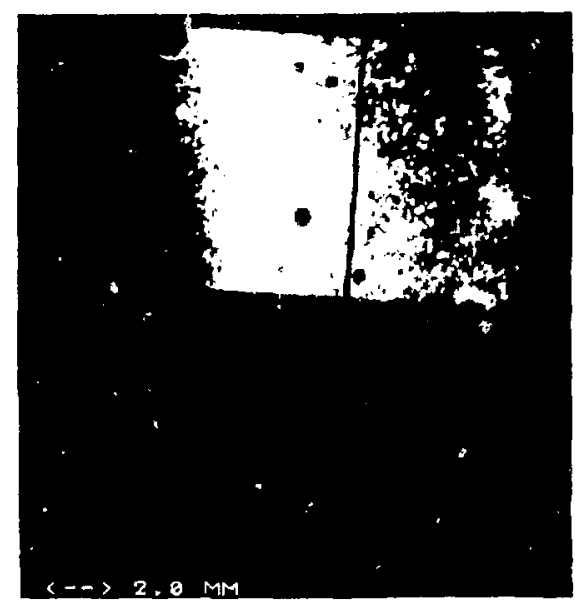

(b)

Figure 5. (a) Wedges of pure $\mathrm{Fe}$ (top) and $\mathrm{Fe}_{2} \mathrm{O}_{3}$ (bottom) powders imaged with $x$-rays of energy $E_{1}$; (b) result of digitally subtracting absorption images taken at energies $E_{2}$ and $E_{3}$. Only the image of the pure Fe matrix remains.

holes, the absolute mass absorption coefficient of the air is accurately reconstructed. The convergence is not quite as good for the $100-\mu \mathrm{m}$ holes, and is quite poor for the $50-\mu \mathrm{m}$ holes. Nevertheless, the $50-\mu \mathrm{m}$ holes are spatially resolved. The reasons for the lack of quantitative detail on the $50-\mu \mathrm{m}$ scale are (1) the object has been undersampled and (2) the counting statistics are not sufficient.

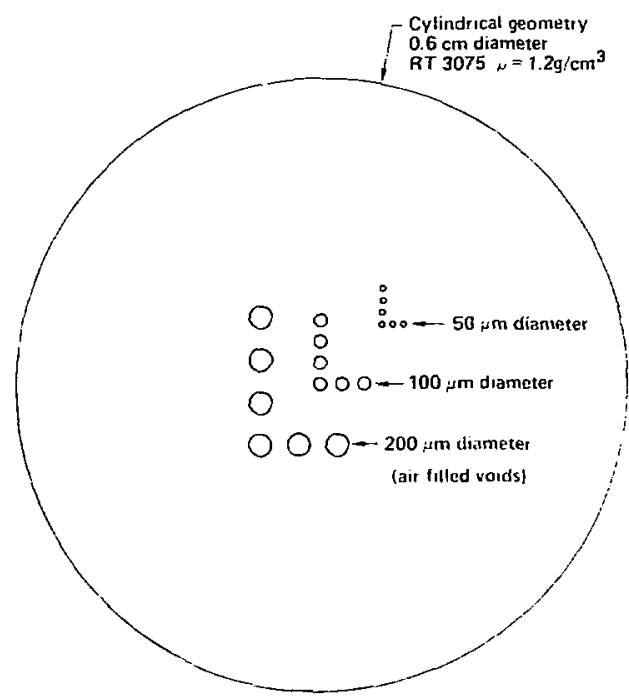

Figure 6. Actual test phantom for demonstrating the high contrast spatial resolution of the CT system.

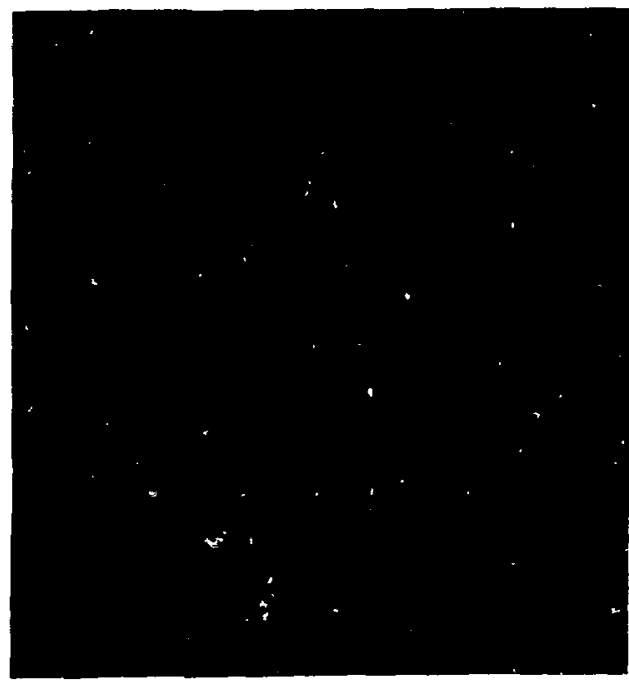

Figure ?. Cross section of test phantom reconstructed from 60 unique projections. The projection data was calculated for this CT system using Monte Carlo miethods.

The suggested sampling rate for reconstruction is given by $P=(\pi / 4)(D / w)$, where $P$ is the number of unique projections required for a given pixel width. For the phantom considered in this example, $P$ should be greater than 180 . Because of computer limitations, however, our sample rate was only a third of this number. The effects of this 
undersampling are apparent in the streak artifacts observed in the reconstructed image. These artifacts can be substantially reduced by taking more projections; however, even with the large undersampling, $50-\mu \mathrm{m}$ features are clearly resolved.

Earlier in this paper, it was demonstrated that an incident $x$-ray fluence of $10^{10}$ photons per unit pixel area was required to achieve a $1 \%$ sensitivity. In the present calculation, an incident fluence of approximately $5 \times 10^{3}$ photons per pixel was used. This low dose could account for :nuch of the lack of contrast in the $50-\mu \mathrm{m}$ holes, although we have yet to clearly study this effect. Also, x-ray scattering from the lowest $z$ phantom would impair the image. This problem could be reduced by moving the detector farther from the sample.

\section{CONCLUSION}

We have demonstrated the potential of SR for imaging studies. We have described experimental apparatus for performing high-resolution tomography, and have included the results of computer calculations which support its effectiveness. The development of high-resolution CT with chemical-state sensitivity could significaritly expand our ability to study microstructures non-destructively.

\section{ACKNOWLEDGEMENT}

We would like to acknowledge the help of C. E. Frerking (LLNL) for his help with zoning the Monte-Carlo problem.

\section{REFERENCES}

1. S. W. Rowland, in Image Reconstruction from Projections, edited by G. T. Herman (SpringerVerlag, New York, 1979), p. 9.

2. G. T. Herman, Image Reconstruction from Projections: The Fundamentals of Computerized Tomography, (Academic Press, New York, 1980).

3. L. Grodzins, Nucl. Inst. and Meth. 206, 541 (1983).

4. U. Bonse et al., Nucl. Inst. and Meth. (in press).

5. R. Aiken, Photometrics, Inc., Tucson, AZ, (private communication), (1986).

6. B. Jacobson, Acta Radicl. 89, 437 (1953).

7. L. Grodzins, Nucl. Inst. and Meth. 206, 547 (1983).

8. G. H. Glover and R. L. Eisner, J. Comp. Ass. Tomography 3, 85 (1979).

9. T. Wilcox and E. Lent, COG (to be published).

10. R. H. Huesman, G. T. Gullberg, W. L. Greenberg, and T. F. Budinger, Donner Algorithms for Reconstruction Tomography, (Lawrence Berkeley Laboratory, University of California, October 1977). 